\title{
ATIVIDADE RESPIRATÓRIA DA MICROBIOTA E CONTEÚDO DE GLICOSE EM RESPOSTA À ADIÇÃO DE FÓSFORO EM SOLO DE CERRADO(1)
}

\author{
Adão de Siqueira Ferreira ${ }^{(2)}$, Reinaldo Silva de Oliveira ${ }^{(3)}$, Maria \\ Amelia dos Santos ${ }^{(4)}$ \& Elias Nascentes Borges ${ }^{(4)}$
}

\begin{abstract}
RESUMO
A atividade metabólica dos microrganismos é um dos principais processos reguladores das transformações de nutrientes no solo. No entanto, a atividade microbiana do solo é influenciada por fatores como a disponibilidade de nutrientes, incluindo o P. Este trabalho teve como objetivo avaliar o efeito da adição de $P$ (de 50 a $500 \mathrm{mg} \mathrm{kg}^{-1}$ de $\mathrm{P}$ no solo) na atividade respiratória da microbiota e nos teores de glicose extra e intracelular em um Latossolo Vermelho distrófico fase Cerrado. As amostras de solo foram avaliadas quanto ao $\mathrm{C}$ liberado $\left(\mathrm{C}-\mathrm{CO}_{2}\right)$ pela atividade microbiana; pelo $\mathrm{C}$ orgânico total e pela glicose extra e intracelular. Durante o período de incubação (31 dias), a adição de $P$ aumentou a atividade respiratória diária de 6,30 para $23,59\left(\mathrm{mg} \mathrm{kg}^{-1} \mathrm{dia}^{-1}\right.$ de $\mathrm{C}-\mathrm{CO}_{2}$ no solo), quando comparado com o controle. No entanto, a relação entre $\mathrm{C}-\mathrm{CO}_{2}$ liberado por dia, por unidade de $\mathrm{P}$ adicionado, diminuiu, mostrando uma redução da eficiência na utilização do $P$ adicionado. O teor de glicose extracelular no solo foi menor do que o encontrado intracelularmente. Ao final de 31 dias de incubação, o teor de glicose intracelular reduziu-se em decorrência da adição de $\mathbf{P}$ no solo, sugerindo maior consumo de glicose pelos microrganismos nas condições de adição do nutriente. Houve correlação negativa $(r=-0,98, p<0,01)$ entre a respiração diária e a glicose intracelular. Aos 31 dias de incubação, o CO total do solo diminuiu com a adição de $500 \mathrm{mg} \mathrm{kg}^{-1}$ de $\mathrm{P}$ no solo. A estreita relação entre o aumento da atividade e a diminuição de glicose intracelular sugere que a resposta da microbiota à adição de $P$ pode estar associada ao conteúdo do açúcar no solo.
\end{abstract}

Termos de indexação: glicose, carbono orgânico, respiração do solo, fósforo, Cerrado.

\footnotetext{
(1) Trabalho financiado pelo Instituto de Ciências Agrárias (ICIAG) da Universidade Federal de Uberlândia - UFU. Recebido para publicação em junho de 2007 e aprovado em junho de 2008.

(2) Professor adjunto II, Microbiologia Agrícola e Ambiental, Universidade Federal de Uberlândia - UFU. CEP 38400-902 Uberlândia (MG). E-mail: adaosferreira@yahoo.com.br

(3) Aluno de Graduação do Curso de Agronomia, Instituto de Ciências Agrárias, UFU. E-mail: reinaldo_agro@yahoo.com.br

(4) Professor associado II, Instituto de Ciências Agrárias, UFU. E-mail: elias@ufu.br
} 


\title{
SUMMARY: RESPIRATORYACTIVITYOF SOIL MICROBIOTAAND GLUCOSE CONTENT IN RESPONSE TO PHOSPHORUS ADDITION IN CERRADO SOIL-BRAZIL
}

\begin{abstract}
Metabolic activity of microorganisms is one of the main processes of control on the nutrient transformation in soil. However, the activity of the soil microbiota is affected by various factors such as the available nutrients, including phosphorus. The present work had as objective to show an approach of the effect of phosphorus addition (50 to $500 \mathrm{mg} \mathrm{kg}^{-1} \mathrm{P}$ of dry soil) on the respiratory response of soil microbiota and the amount of extra and intracellular glucose in typic acrustox of Cerrado.The following analyses were carried: amount of $\mathrm{C}-\mathrm{CO}_{2}$ evolved; total organic carbon e; extra and intracellular glucose in soil. During the incubation for 31 days, the respiratory activity (daily activity) increased in 6,30 to 23,59 with phosphorus addition, when compared to the control. However, the ratio between the daily carbon evolved $\left(\mathrm{C}-\mathrm{CO}_{2}\right)$ and the added phosphorus decreased, showing a reduction of the efficiency of nutrient utilization. The amount of the extracellular glucose was very smaller than the intracellular glucose. At end of 31 days of incubation, the amount of glucose decreased due to phosphorus addition, showing the consumption of sugar by microorganisms under experimental conditions. Negative metabolic correlation $(r=-0.97, p<0.01)$ between the daily respiration and the intracellular glucose was shown. The soil organic carbon decreased only in the treatment with $500 \mathrm{mg} P \mathrm{~kg}$ ${ }^{1}$ of dry soil, being other treatments similar to the control. The strong relation between an increasing of microorganism activity and a decreasing of intracellular glucose occurred, suggesting that the response of soil microbiota to phosphorus addition can be associated to the intracellular glucose content.
\end{abstract}

Index terms: Glucose, organic carbon, soil respiration, phosphorus, Cerrado.

\section{INTRODUÇÃO}

O metabolismo microbiano é um dos principais processos reguladores da transformação de nutrientes no solo. Entre as formas de avaliação da atividade metabólica da microbiota do solo, destaca-se a quantificação de $\mathrm{C}$ pela liberação de $\mathrm{CO}_{2}$, conhecida por respiração do solo (Stotzky, 1965). No entanto, a atividade dos microrganismos está estritamente relacionada com fatores ambientais, como temperatura, umidade, $\mathrm{pH}$, matéria orgânica e nutrientes (Stotzky \& Norman, 1961; Atlas \& Bartha, 1993). Em particular, o N e o $\mathrm{P}$ são os principais nutrientes exigidos no metabolismo devido às demandas estruturais e funcionais das células (Sylvia et al., 1999).

Vários pesquisadores têm relatado que o $\mathrm{P}$ é o principal nutriente limitante no solo devido a sua baixa disponibilidade (Ilstedt \& Singh, 2005; Gnankambary et al., 2008). Os estudos focalizaram inicialmente as informações sobre a aplicação de P no solo com a produção de matéria seca das plantas, sendo poucos os associados à resposta metabólica da microbiota do solo (Nziguheba et al., 1988; Cleveland et al., 2002; Ilstedt et al., 2003). Além disso, sabe-se que a atividade dos microrganismos depende da quantidade e qualidade da matéria orgânica do solo e da disponibilidade de nutrientes (Cleveland et al., 2002; Ilstedt et al., 2007; Gnankambary et al., 2008).
Atualmente, relatos importantes têm sido feitos a respeito da necessidade de se conhecer a resposta metabólica (liberação de $\mathrm{CO}_{2}$ ) do solo em decorrência da preocupação com o aquecimento global. Trabalhos, como de Edward et al. (2007) e Mondini et al. (2007), têm mostrado a importância da adição de nutrientes no solo com relação à liberação de $\mathrm{CO}_{2}$ dióxido de $\mathrm{C}$. Além disso, o estudo das exigências nutricionais dos microrganismos deve ser prioridade nas relações dos processos biológicos nos ecossistemas, a exemplo do $\mathrm{P}$ (Porder et al., 2007; Qiu et al., 2007; Gnankambary et al., 2008).

Este trabalho teve como objetivo avaliar o efeito da adição de $\mathrm{P}$ na resposta respiratória da microbiota do solo e nos teores de glicose extracelular e intracelular.

\section{MATERIAL E MÉTODOS}

A pesquisa foi realizada com amostra de um Latossolo Vermelho distrófico localizado na Fazenda Experimental (Fazenda do Glória), em área pertencente ao Instituto de Ciências Agrárias da Universidade Federal de Uberlândia, em janeiro de 2007. A amostra foi obtida no período das chuvas, na região de Uberlândia. Na área, a vegetação predominante era de gramíneas com destaque para as espécies 
pertencentes ao gênero Brachiaria. Subamostras foram realizadas numa área de $600 \mathrm{~cm}^{2}(30 \times 20 \mathrm{~cm})$ por $10 \mathrm{~cm}$ de profundidade.

No laboratório, a amostra de solo foi passada em peneira com malha de $3,35 \mathrm{~mm}$. Uma subamostra de $100 \mathrm{~g}$ foi seca em estufa a $70{ }^{\circ} \mathrm{C}$ por $24 \mathrm{~h}$. O solo seco foi triturado em cadinho de porcelana e enviado ao Laboratório de Análises de Solos (ICIAG-UFU) para caracterização química (Quadro 1). Foram feitas ainda caracterizações físicas, determinando-se umidade conforme Tedesco et al. (1995). A amostra de solo em condições naturais ( $\mathrm{SCN}$ ) foi mantida na geladeira $\left(4^{\circ} \mathrm{C}\right)$ até o dia seguinte, quando foi montado o experimento com $P$.

Os tratamentos com $\mathrm{P}$ foram instalados em frascos $(1 / 2 \mathrm{~L})$ de vidro com tampa de vedação. Utilizou-se como fonte de $\mathrm{P}$ uma solução de $\mathrm{Na}_{2} \mathrm{HPO}_{4} .7 \mathrm{H}_{2} \mathrm{O}$ em água destilada. Uma alíquota da solução foi adicionada ao solo, acondicionado nos frascos de incubação (100 g de SCN) correspondentes de forma a obter 50, 100, 200, 300, 400 e $500 \mathrm{mg} \mathrm{kg}^{-1}$ de $\mathrm{P}$ no solo seco, em duas repetições. O tratamento controle recebeu água destilada e a umidade do solo foi corrigida a $22 \%\left(\mathrm{p} \mathrm{p}^{-1}\right)$ em todos os tratamentos. A temperatura ambiente, durante a realização do experimento de atividade microbiana, variou de 21,5 a $24{ }^{\circ} \mathrm{C}$, com temperatura média de $23^{\circ} \mathrm{C}$.

Quadro 1. Caracterização física, química e biológica do solo ${ }^{(1)}$

\begin{tabular}{|c|c|}
\hline Caracterização & Quantidade \\
\hline Areia $\left(\mathrm{g} \mathrm{kg}^{-1}\right)$ & 550 \\
\hline Argila $\left(\mathrm{g} \mathrm{kg}^{-1}\right)$ & 420 \\
\hline Silte $\left(\mathrm{g} \mathrm{kg}^{-1}\right)$ & 30 \\
\hline Densidade $\left(\mathrm{mg} \mathrm{m}^{-3}\right)$ & 1,41 \\
\hline Capacidade de retenção de água $\left(\mathrm{g} \mathrm{kg}^{-1}\right)$ & 260 \\
\hline Umidade $(\%)^{(2)}$ & 9,98 \\
\hline $\mathrm{pH}$ (água) & 5,6 \\
\hline $\mathrm{P}\left(\mathrm{mg} \mathrm{dm}{ }^{-3}\right)$ & 4,8 \\
\hline $\mathrm{K}\left(\mathrm{mg} \mathrm{dm^{-3 }}\right)$ & 11,0 \\
\hline $\mathrm{Ca}\left(\mathrm{cmol}_{\mathrm{c}} \mathrm{dm}^{-3}\right)$ & 1,1 \\
\hline $\mathrm{Mg}\left(\mathrm{cmol}_{\mathrm{c}} \mathrm{dm}^{-3}\right)$ & 0,7 \\
\hline $\mathrm{Al}\left(\mathrm{cmol}_{\mathrm{c}} \mathrm{dm}^{-3}\right)$ & 0,0 \\
\hline $\mathrm{Al}+\mathrm{H}\left(\mathrm{cmol}_{\mathrm{c}} \mathrm{dm}^{-3}\right)$ & 3,1 \\
\hline Carbono orgânico ( $\mathrm{g} \mathrm{kg}^{-1}$ de solo) & 11,1 \\
\hline Glicose $\left(\mathrm{mg} \mathrm{kg}^{-1}\right.$ de solo) ${ }^{(3)}$ & $<0,01$ \\
\hline Respiração basal (mg C- $\mathrm{CO}_{2} \mathrm{~kg}^{-1}$ de solo dia $\left.{ }^{-1}\right)^{(4)}$ & 5,68 \\
\hline
\end{tabular}

${ }^{(1)}$ Amostra de solo coletada a $10 \mathrm{~cm}$ de profundidade. Solo classificado como Latossolo Vermelho distrófico. ${ }^{(2)}$ Umidade determinada no mesmo dia da coleta $\left(\mathrm{g} \mathrm{g}^{-1}\right.$ de solo seco). ${ }^{(3)}$ Determinada no dia da coleta do solo segundo o procedimento descrito no item Material e Métodos deste trabalho. ${ }^{(4)}$ Respiração determinada num período de incubação de oito dias em temperatura ambiente e com $60 \%$ de umidade em relação à capacidade de campo do solo.
A atividade microbiana foi avaliada pela quantificação do $\mathrm{CO}_{2}$ liberado, conforme descrito por Stotzky (1965), com modificações. Copos descartáveis (40 mL) contendo $10 \mathrm{~mL}$ de hidróxido de sódio $1 \mathrm{~mol} \mathrm{~L}^{-1}$ foram colocados dentro de cada frasco de incubação para a captura de $\mathrm{CO}_{2}$. Os frascos foram hermeticamente fechados e mantidos em temperatura ambiente por 31 dias. A determinação do $\mathrm{CO}_{2}$ liberado foi realizada no terceiro, décimo, décimo oitavo e trigésimo primeiro dias após a instalação do experimento. Após cada período de incubação, foram retirados os copos plásticos dos frascos e, nestes, colocados $5 \mathrm{~mL}$ de $\mathrm{BaCl}_{2} \cdot 2 \mathrm{H}_{2} \mathrm{O} 1 \mathrm{~mol} \mathrm{~L}^{-1}$ e algumas gotas de fenoftaleína $1 \%$, sendo o excesso de $\mathrm{NaOH}$ titulado com $\mathrm{HCl}$ 0,5 mol L-1. Novos copos plásticos com $\mathrm{NaOH}$ foram colocados nos frascos de vidro para subseqüentes períodos de incubação. A amostra do branco foi constituída em frasco sem a porção de solo. A quantidade de $\mathrm{C}$ liberada, em $\mathrm{mg} \mathrm{kg}^{-1}$ de $\mathrm{C}-\mathrm{CO}_{2}$ no solo seco, foi calculada pela fórmula:

$$
\mathrm{QC}=\left[(\mathrm{B}-\mathrm{A}) \times \mathrm{M}_{\mathrm{c}} \times \mathrm{M}_{\mathrm{HCl}}\right] / \mathrm{M}_{\mathrm{s}} \times 2
$$

sendo $\mathrm{QC}=$ quantidade de $\mathrm{C}$ liberada pelos microrganismos, em mg kg-1 de $\mathrm{C}-\mathrm{CO}_{2}$ no solo; $\mathrm{B}=$ volume de $\mathrm{HCl}$ necessário para titular o excedente de $\mathrm{NaOH}$ da prova em branco; $\mathrm{A}=$ volume de $\mathrm{HCl}$ necessário para titular o excedente de $\mathrm{NaOH}$ da amostra; $\mathrm{M}_{\mathrm{c}}=$ massa atômica do $\mathrm{C}(12) ; \mathrm{M}_{\mathrm{HCl}}=$ molaridade da solução de $\mathrm{HCl} 0,5 \mathrm{~mol} \mathrm{~L}^{-1} ; \mathrm{M}_{\mathrm{s}}=$ massa seca do solo incubado (em kg); 2: proporcionalidade de massa na reação entre o $\mathrm{C}$ liberado, na forma de $\mathrm{CO}_{2}$, e o consumo de $\mathrm{NaOH}$ (Stotzky, 1965).

As análises de $\mathrm{CO}$ total foram feitas no início do experimento (Quadro 1) e após 31 dias de incubação em $2 \mathrm{~g}$ de solo seco, conforme Tedesco et al. (1995). Antes da análise, o solo foi homogeneizado e $20 \mathrm{~g}$ foram secos a $70^{\circ} \mathrm{C}$ por $48 \mathrm{~h}$.

A glicose foi avaliada pelo método enzimático conforme descrito no manual de instruções do fabricante (LABORLAB, Brasil). O método é usado para determinar o nível de glicose no soro e plasma (Henry et al., 1974), sendo feita uma adaptação para determinação do açúcar no solo. A glicose extracelular foi determinada em $20 \mathrm{~g}$ de SCN, sendo a porção transferida para Erlenmeyer $(125 \mathrm{~mL})$ contendo $\mathrm{NaCl}$ $20 \mathrm{~mL}$ de $0,5 \%$. A solução foi agitada por $20 \mathrm{~min}$ em temperatura ambiente. A glicose intracelular foi determinada utilizando a mesma porção de solo, lisando as células microbianas pela irradiação no forno microondas por 2 min (Ferreira et al., 1999). Em seguida, as células foram agitadas por $20 \mathrm{~min}$ em banho-maria $\left(70{ }^{\circ} \mathrm{C}\right)$. As soluções das duas frações foram filtradas em papel-de-filtro quantitativo $(8 \mu \mathrm{m})$ e centrifugadas a $6.000 \mathrm{~g}$ por $10 \mathrm{~min}$.

Uma alíquota de $500 \mu \mathrm{L}$ de cada sobrenadante foi transferida para tubo de ensaio contendo $2,5 \mathrm{~mL}$ de reativo enzimático (volume total de $3 \mathrm{~mL}$ ). A dosagem 
de glicose no extrato foi determinada com auxílio de uma curva-padrão de glicose contendo 0, 5, 10, 50 e $100 \mu \mathrm{g}$ em $3 \mathrm{~mL}$. Após $12 \mathrm{~min}$ de reação, foi feita a leitura em espectrofotômetro a $505 \mathrm{~nm}$. Os resultados foram calculados de forma a obter o teor específico de glicose por unidade de solo seco, com o ajuste de umidade das amostras. A dosagem de glicose foi determinada no início do experimento (Quadro 1) e no final de 31 dias de incubação.

O experimento foi realizado segundo um delineamento inteiramente casualizado. Os resultados obtidos foram submetidos à análise de regressão, e as médias comparadas por teste de médias, quando necessário. Análises de correlação foram feitas entre variáveis para avaliar o grau de associação das informações obtidas a $5 \%$. Os resultados de atividade microbiana do solo, quanto à utilização do $\mathrm{P}$, foram avaliados pela equação de Michaelis-Menten (White, 2007):

$$
\mathrm{A}=\left(\mathrm{A}_{\max } \mathrm{P}\right) /\left(\mathrm{k}_{\mathrm{m}}+\mathrm{P}\right)
$$

sendo $\mathrm{A}=$ atividade microbiana $\left(\mathrm{mg} \mathrm{kg}^{-1} \mathrm{dia}^{-1} \mathrm{de} \mathrm{C}\right.$ $\mathrm{CO}_{2}$ no solo); $\mathrm{A}_{\max }=$ atividade máxima resultante da adição de $\mathrm{P}$ no solo; $\mathrm{P}=\mathrm{P}$ adicionado no solo $\left(\mathrm{mg} \mathrm{kg}^{-1}\right.$ de $\mathrm{P}) ; \mathrm{k}_{\mathrm{m}}=$ constante de Michaelis-Menten (mg kg-1 de $\mathrm{P}$ no solo) em relação à utilização de $\mathrm{P}$ e à resposta da atividade microbiana.

\section{RESULTADOS}

A adição de $\mathrm{P}$ afetou a atividade metabólica dos microrganismos no solo, indicada pela liberação de $\mathrm{CO}_{2}$ (Figura 1). Em todos os tratamentos, as

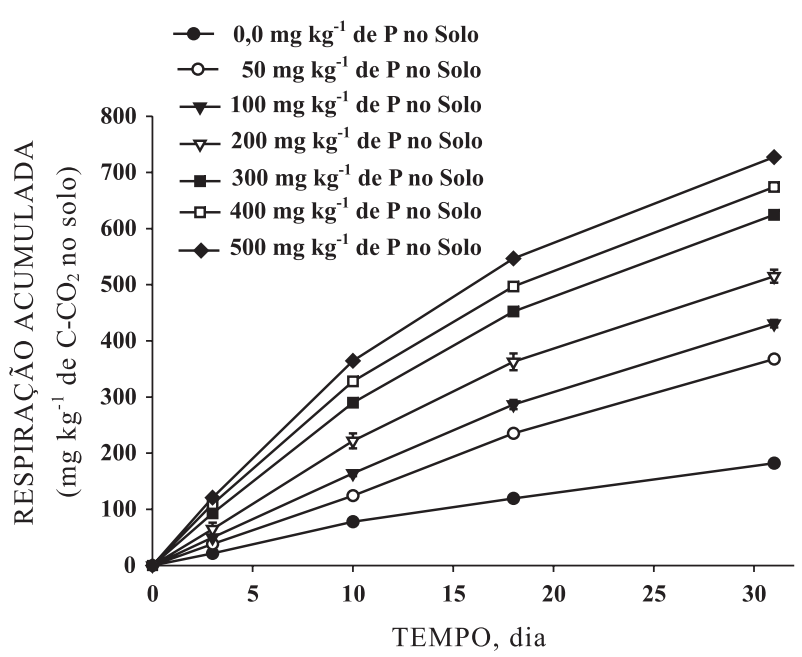

Figura 1. Liberação de $\mathrm{CO}_{2}$ do solo em resposta à adição de fósforo. As barras indicam os intervalos de confiança a $5 \%(n=2)$. diferenças de atividade com adição de $\mathrm{P}$ foram significativas, quando comparadas ao controle. Essas aumentaram no decorrer do período de incubação. A maior atividade foi verificada no tratamento com $500 \mathrm{mg} \mathrm{kg}^{-1}$ de P no solo.

Os resultados mostraram que a taxa diária de liberação de $\mathrm{C}-\mathrm{CO}_{2}$ nos tratamentos com $\mathrm{P}$ aumentou de 6,30 para 23,59 (mg kg-1 dia $^{-1}$ de C- $\mathrm{CO}_{2}$ no solo) em relação ao tratamento controle. A equação de regressão, segundo o modelo de Mechaelis-Menten, mostrou excelente grau de ajuste (Figura 2a). Em função da adição de $\mathrm{P}$, a resposta da atividade permitiu obter um valor crítico de utilização de $\mathrm{P}$ (319 mg kg-1 de $\mathrm{P}$ no solo). $\mathrm{O}$ valor máximo de atividade calculado foi de $37,8 \mathrm{mg} \mathrm{kg}^{-1} \mathrm{dia}^{-1}$ de C-CO $\mathrm{CO}_{2}$ no solo. Entretanto, as relações entre taxa de liberação diária de $\mathrm{CO}_{2}$ por unidade de $\mathrm{P}$ adicionado diminuíram quando da adição do nutriente. Um elevado valor de $\mathrm{R}^{2}$ foi obtido para a equação de regressão dessa relação em conseqüência da adição de $\mathrm{P}$ (Figura $2 \mathrm{~b}$ ). Utilizando essa equação para o valor de $319 \mathrm{mg} \mathrm{kg}^{-1}$ de $\mathrm{P}$ no solo, obtém-se uma relação de 0,060 de C- $\mathrm{CO}_{2}$ por $\mathrm{P}$ adicionado.
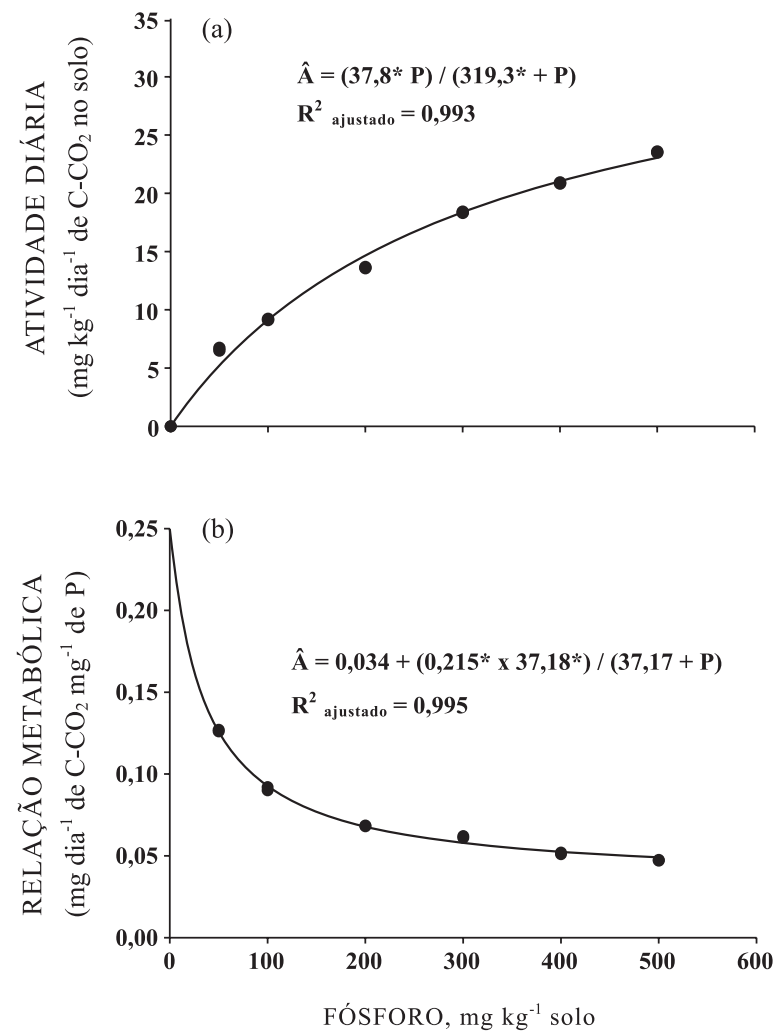

Figura 2. Equações de regressão da atividade metabólica em função da adição de fósforo no solo. (a) regressão do modelo Michaelis-Menten, entre respiração diária e adição de $P$. (b) regressão entre relação metabólica (taxa diária por fósforo adicionado) e adição de $P$. * significativo a $1 \%$. 
Os tratamentos com 0 a $400 \mathrm{mg} \mathrm{kg}^{-1}$ de $\mathrm{P}$ no solo não diferiram quanto ao total de $\mathrm{C}$ no solo (Quadro 2). Entretanto, no tratamento com a maior dose de $\mathrm{P}$, foi observada uma redução significativa do C orgânico total no final do período de incubação, quando comparado com os demais tratamentos. E importante ressaltar que o período de incubação (31 dias) foi muito curto para esperar reduções mais expressivas de $\mathrm{C}$ orgânico total no solo.

Os teores de glicose extracelular e intracelular variaram de menos de 0,01 a $15,90 \mathrm{mg} \mathrm{kg}^{-1}$ (Quadro 2). O teor de glicose extracelular foi muito abaixo ao encontrado intracelularmente. Além disso, a adição de $\mathrm{P}$ ao solo levou a um maior consumo de glicose pelos microrganismos, diminuindo os valores do açúcar intracelular no solo.

Uma alta correlação negativa $(r=-0,98)$ ocorreu entre glicose intracelular e taxa diária de liberação de $\mathrm{CO}_{2}$, mostrando que a glicose no solo diminui com o aumento da atividade microbiana (Figura 3). A adição de $\mathrm{P}$ acima de $300 \mathrm{mg} \mathrm{kg}^{-1}$ de $\mathrm{P}$ no solo teve como conseqüência redução expressiva de glicose intracelular no solo.

\section{DISCUSSÃO}

Os resultados demonstram que o $\mathrm{P}$ influencia a atividade metabólica da microbiota do solo. Relatos na literatura, como os de Saggar et al. (1998), Demetz \& Insam (1999), Cleveland et al. (2002) e Porder et al. (2007), têm mostrado a importância metabólica do P sobre a atividade de microrganismos, destacando que este elemento pode ser o principal fator limitante na ciclagem de nutrientes no solo. Em solos tropicais, essa limitação ocorre, principalmente, devido à tendência de adsorção de aniôn fosfato em compostos de óxidos de $\mathrm{Al}$ e $\mathrm{Fe}$, predominante em grupos funcionais de superfície de caulinita em Latossolos

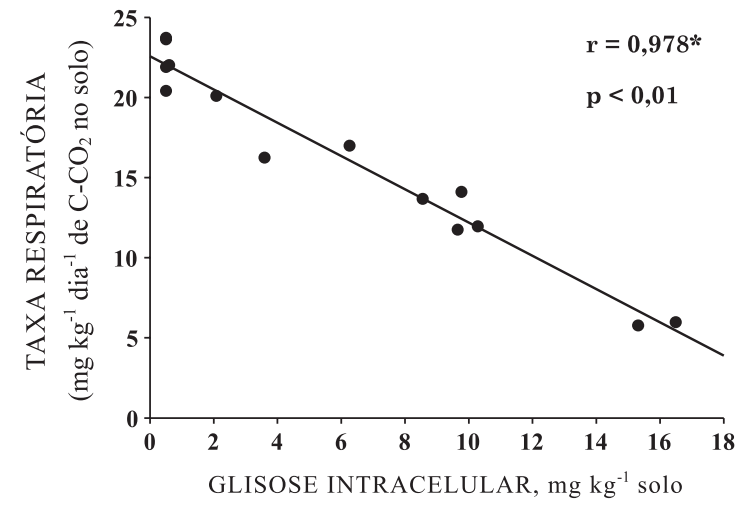

Figura 3. Correlação metabólica entre o teor de glicose intracelular e a taxa respiratória do solo. * correlação significativa a $1 \%(n=14)$.

(Sanchez, 2002; Ilstedt \& Singh, 2005), diminuindo, assim, a disponibilidade do nutriente na fração lábil (Ilstedt et al., 2003; Ilstedt \& Singh, 2005).

O metabolismo microbiano está associado a vários processos químicos e biológicos no solo, devendo-se buscar novas formas de avaliação dos atributos envolvidos na resposta bioquímica do solo. As avaliações da resposta metabólica da microbiota mostraram comportamento diferencial em decorrência da adição de $\mathrm{P}$, destacando-se a aplicação de até $319 \mathrm{mg} \mathrm{kg}^{-1}$ de P no solo como limite de proporcionalidade de resposta metabólica. Deve-se destacar que o efeito de outros fatores reguladores do metabolismo não foi avaliado. Vários autores têm destacado que outros fatores além do P são importantes, a exemplo do C e de N (Howard \& Howard, 1993; Schmidt et al., 1999; Teklay et al., 2006; Gnankambary et al., 2008), quando a umidade e a temperatura forem constantes.

$\mathrm{A}$ adição de $\mathrm{P}$ levou a um decréscimo no teor de glicose intracelular, sugerindo o consumo do açúcar pelos microrganismos. O conteúdo de glicose no solo pode ter sido um dos fatores limitantes da resposta

Quadro 2. Teores de carbono orgânico e glicose em decorrência da adição de fósforo no solo $(\mathrm{n}=2)$

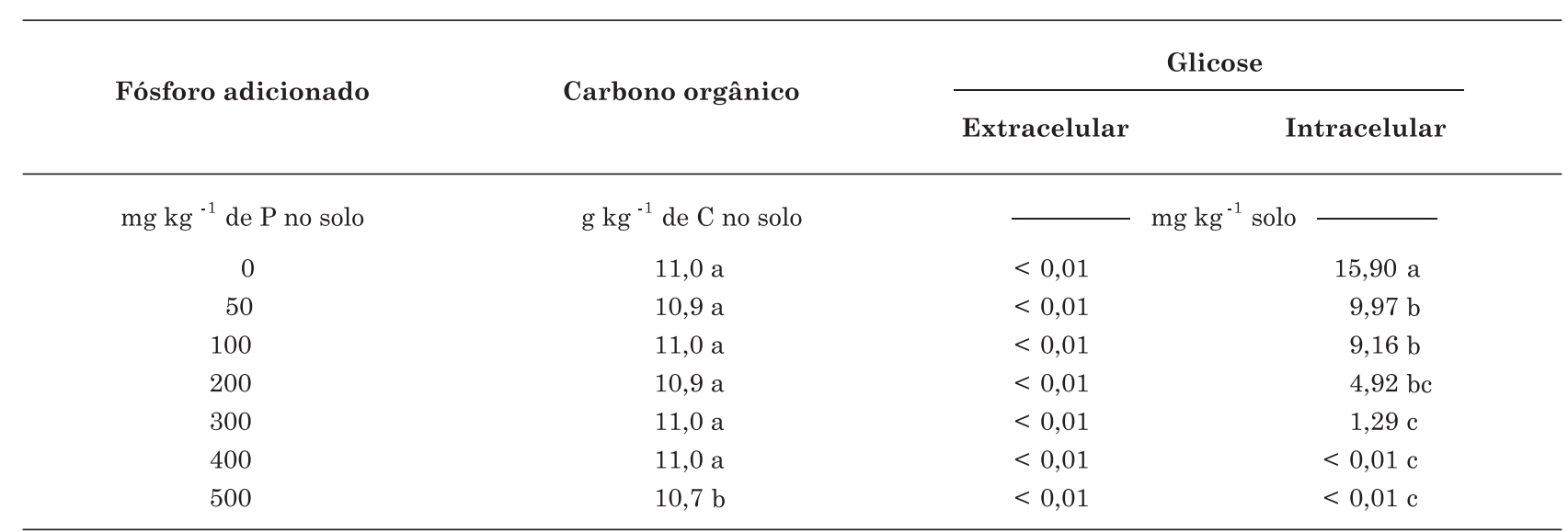

Letras minúsculas diferentes indicam diferenças significativas entre tratamentos pelo teste de Tukey a $1 \%$. 
metabólica ao $\mathrm{P}$, principalmente quando a adição foi acima de $319 \mathrm{mg} \mathrm{kg}^{-1}$ de $\mathrm{P}$ no solo. Isso pode ser justificado pela coincidência entre a resposta metábolica (Figura 2) e o decréscimo expressivo de glicose no solo (Quadro 2). Além disso, a estreita relação entre o aumento da atividade e a diminuição de glicose intracelular (Figura 3) sugere que a resposta da microbiota do solo à adição de $\mathrm{P}$ pode estar associada ao conteúdo do açúcar no solo. Contudo, o teor de glicose pode estar associado, em parte, à qualidade da matéria orgânica e sua transformação, como destacado por Cleveland et al. (2002), Ilstedt \& Singh (2005) e Gnankanbary et al. (2008). Cleveland et al. (2002) relataram a importância da adição de glicose e P na avaliação da atividade da microbiota do solo, sugerindo a relação $\mathrm{C} / \mathrm{P}$ como fator de grande relevância na regulação dos processos biológicos do solo.

O teor de glicose pode ser um importante atributo bioquímico resultante da atividade metabólica dos microrganismos no solo. A redução de glicose intracelular pode estar associada a demandas metabólicas decorrentes da adição de P, a saber: (1) ao catabolismo para a produção de energia, com a liberação de $\mathrm{CO}_{2} ;(2)$ a necessidade anabólica para a síntese estrutural das células; e (3) a síntese de compostos de reserva, como glicogênio (Sylvia et al., 1999). No entanto, pouco se conhece a respeito do metabolismo do solo quanto à demanda de carboidratos associados a estímulos de fatores abióticos, a exemplo do P.

Estudos envolvendo os processos biológicos e a disponibilidade de nutrientes no solo, em especial aqueles que limitam a atividade microbiana, têm sido relatados como base de ampliação dos conhecimentos teóricos, fornecendo suporte à aplicação prática de estratégia de manejo dos solos (Teklay et al., 2006; Zheng et al., 2007; Gnankambary et al., 2008). Assim, trabalhos que envolvem as demandas nutricionais da microbiota, associadas ao potencial dos solos quanto à quantidade e qualidade de matéria orgânica, devem ser valorizados em sistemas agrícolas do Cerrado.

\section{CONCLUSÕES}

1. A adição de $\mathrm{P}$ aumentou a atividade respiratória avaliada pelo $\mathrm{C}$ liberado, quando comparado com a do tratamento-controle, porém, o aumento da atividade não foi proporcional à quantidade adicionada do nutriente.

2. O teor de glicose intracelular foi maior em comparação ao do nível extracelular. A adição de $\mathrm{P}$ diminuiu significativamente os teores de glicose intracelular no período de 31 dias de incubação em relação ao do controle.

3. A estreita relação entre o aumento da atividade respiratória e a diminuição de glicose intracelular sugere que a resposta da microbiota do solo à adição de $\mathrm{P}$ pode estar associada ao conteúdo de glicose intracelular.

\section{AGRADECIMENTOS}

Os autores agradecem aos Laboratórios de Manejo do Solo e Microbiologia Agrícola e Ambiental pelo apoio financeiro na realização dos experimentos.

\section{LITERATURA CITADA}

ALTAS, R.M. \& BARTHA, R. Microbial ecology: Fundamentals and applications. 3.ed. NewYork, The BenjaminCummings Publishing Company, 1993. 563p.

CLEVELAND, C.; TOWNSEND, A.R. \& SCHMIDT, S.K. Phosphorus limitation of microbial processes in moist tropical forest: Evidence from short-term laboratory incubations and field studies. Ecosystems, 5:680-691, 2002.

DEMETZ, M. \& INSAM, H. Phosphorus availability in a forest soil determined with a respiratory assay compared to chemical methods. Geoderma, 89:259-271, 1999.

EDWARD, C.C.; HAYES, M.H.B. \& CIAVATTA, C. Organic wastes in soils: Biogeochemical and environmental aspects. Soil Biol. Biochem., 39:1239-1243, 2007.

FERREIRA, A.S.; CAMARGO, F.A.O. \& VIDOR, C. Utilização de microondas na avaliação da biomassa microbiana do solo. R. Bras. Ci. Solo, 23:991-996, 1999.

GNANKAMBARY, Z.; ILSTEDT, U.; NYBERG, G.; HIEN, V. \& MALMER, A. Nitrogen and phosphorus limitation of soil microbial respiration in two tropical agroforestry parklands in the south-Sudanese zone of Burkina Faso: The effects of canopy and fertilization. Soil Biol. Biochem., 40:350-359, 2008.

HENRY, R.J.; CANNON, D.C. \& WINKELMAN, J. Clinical chemistry principies and techniques. 2 ed. NewYork, Harper and Row Publishers, 1974. p.1288.

HOWARD, D.M. \& HOWARD, P.J.A. Relationships between $\mathrm{CO}_{2}$ evolution, moisture content and temperature for a range of soil types. Soil. Biol. Biochem., 25:1537-1546, 1993.

ILSTEDT, U.; GIESLER, R.; NORDGREN, A. \& MALMER, A. Changes in soil chemical and microbial properties after a wildfire in a tropical rainforest in Sabah, Malaysia. Soil Biol. Biochem., 35:1071-1078, 2003.

ILSTEDT, U. \& SINGH, S. Nitrogen and phosphorus limitations of microbial respiration in a tropical phosphorus-fixing Acrisol (Ultisol) compared with organic compost. Soil Biol. Biochem., 37:1407-1410, 2005. 
ILSTEDT, U.; SINGH, S. \& NORDGREN, A. Using perlite as a substrate carrier for measuring microbial avalilable phosphorus by respiration kinetics in soils. Biol. Fert. Soil, 43:503-510, 2007.

MONDINI, C.; CAYUELA, M.L.; SINICCO, T.; CORDARO, A.R. \& SÁNCHEZ-MONEDERO, M.A. Greenhouse gas emissions and carbon sink capacity of amended soil evaluated under laboratory conditions. Soil. Biol. Biochem., 39:1366-1374, 2007.

NZIGUHEBA, G.; PALM, C.A.; BURESH, R.J. \& SMITHSON, P.C. Soil phosphorus factions and adsorption as affected by organic and inorganic sources. Plant Soil, 198:159168,1988

PORDER, S.; VITOUSEK, P.M.; CHADWICK, O.A.; CHAMBERLAIN, C.P. \& HILLEY, G.E. Uplift, erosion, and phosphorus limitation in terrestrial ecosystems. Ecosystems, 10:159-171, 2007.

QIU, S.; McCOMB, A.J. \& BELL, R.W. A mass-balance approach to measuring microbial uptake and pools of phosphorus in nutrient-amemded soils. Soil Biol. Biochem., 39:187-193, 2007

SAGGAR, S.; PARFITT, R.L.; SALT, G. \& SKINNER, M.F. Carbon and phosphorus transformations during decomposition of pine forest floor with different phosphorus status. Biol. Fert. Soils, 27:197-204, 1998.

SANCHEZ, P.A. Soil fertility and hunger in Africa. Science, 295:2019-2020, 2002.
SCHMIDT, I.K.; JONASSON, S. \& MICHELSEN, A. Mineralization and microbial immobilization of $\mathrm{N}$ and $\mathrm{P}$ in Arctic soil in relation to season, temperature and nutrient amendment. Appl. Soil. Ecol., 11:147-160, 1999.

STOTZKY, G. Microbial respiration. In: BLACK, C.A., ed. Methods of soil analysis. Madison, American Society of Agronomy, 1965. v.2. p.1550-1570.

STOTZKY, G. \& NORMAN, A.G. Factors limiting microbial activities in soil: I. The level of substrate, nitrogen, and phosphorus. Arch. Microbiol., 40:341-369, 1961.

SYLVIA, D.M.; FUHRMANN, J.J.; HARTEL, P.G. \& ZUBERER, D.A. Principles and applications of soil microbiology. New Jersey, Prentice Hall, 1999. 550p.

TEDESCO, M.J.; BOHNEM, H.; GIANELLO, C.; BISSANI, C.A. \& VOLKWEISS, S.J. Análise de solo, plantas e outros materiais. 2.ed. Porto Alegre, Universidade Federal do Rio Grande do Sul, 1995. 174p., (Boletim Técnico, 5)

TEKLAY, T.; NORDGREN, A. \& MALMER, A. Soil respiration characteristics of tropical soils from agricultural and forestry land-uses at Wond Genet (Ethiopia) in response to $\mathrm{C}, \mathrm{N}$ and $\mathrm{P}$ amendments. Soil Biol. Biochem., 38:125133, 2006.

WHITE, D. The physiology and biochemistry of prokaryotes . 3.ed. New York, Oxford University Press, 2007. 628p.

ZHENG, S.; YAO, J.; ZHAO, B. \& YU, Z. Influence of agricultural practices on soil microbial activity measured by microcalorimetry. Eur. J. Soil Biol., 43:1-7, 2007. 